\title{
An unusual neurologic presentation of pediatric neuroinvasive West Nile virus infection: ophthalmoplegia
}

\author{
Senem Ayça ${ }^{1 \oplus}$, Gülşen Akkoç²๑ ${ }^{2}$ Halit Özdemir ${ }^{3 \odot}$, Hatice Nilgün Selçuk Duru ${ }^{4 \oplus}$ \\ Departments of ${ }^{1}$ Paediatric Neurology, ${ }^{2}$ Paediatric Infectious Diseases, ${ }^{3}$ Family Medicine and ${ }^{4}$ Child Health and Diseases, Haseki \\ Training and Research Hospital, İstanbul, Turkey.
}

\begin{abstract}
Background. West Nile virus (WNV) is an uncommon arbovirus infection and is usually asymptomatic in pediatric patients and due to its rarity is not very well known by clinicians.

Case. We present a 5-year-old girl admitted to the Pediatric Emergency Service with fever, vomiting, neck stiffness, walking difficulty and sudden deviation of eyes who was diagnosed with a neuroinvasive WNV infection.

Conclusions. Ophthalmoplegia is an unusual presentation of neuroinvasive WNV and there are no published pediatric cases with ophthalmoplegia in the literature.
\end{abstract}

Key words: West Nile Virus, ophthalmoplegia, ataxia, children.

West Nile virus (WNV) is an uncommon arbovirus infection and is usually asymptomatic in pediatric patients. Infections due to WNV have increased in frequency and include previously virus-free regions, especially since $2018 .^{1}$ Some European countries including Balkan countries and Turkey had higher numbers of reported WNV cases than the previous years. ${ }^{2}$

Neuroinvasive WNV infection presentations include aseptic meningitis, meningoencephalitis and acute flaccid paralysis syndrome. Brainstem encephalitis, cerebellitis, movement disorders, cranial neuropathies, polyneuropathy/radiculopathy, chorioretinitis and optic neuritis are also recognized WNV neurological presentations. ${ }^{3}$ Knowledge of WNS infections and atypical neurological presentations is important for pediatricians and neurologists because of the increased number of cases. Herein, we present a case of pediatric neuroinvasive WNV with ophthalmoplegia,

$\triangle$ Senem Ayça

senemkaleci85@gmail.com

Received 19th May 2020, revised 30th December 2020, accepted 9th January 2021. which is an unusual presentation. To the best of our knowledge, this is the first published pediatric case with ophthalmoplegia in the literature.

\section{Case Report}

A previously healthy 5-year-old girl with fever, vomiting, fatigue, and myalgia presented to a pediatric clinic in September 2019. She was prescribed amoxicillin and antipyretics. A day after admission, additional symptoms such as frontal headache, deviation of eyes, meaningless speech, and walking imbalance progressed and she was admitted to our tertiary hospital in Istanbul, Turkey. Her medical history was uneventful. She was born as a child of nonconsanguineous parents through spontaneous vaginal birth. Her parents recalled her being bitten by mosquitoes a few weeks ago in Esenler/İstanbul.

On admission, she was lethargic with a $39.2^{\circ} \mathrm{C}$ body temperature, and a truncal maculopapular rash was observed. She had isochoric pupils with normal light reflexes, but she had a sudden medial deviation of bilateral eyes and she had 
limited abduction paresis of the bilateral eyes. She had deep tendon reflexes with normal response and her muscle strength was normal. Her plantar reflex was flexor bilaterally. She had neck stiffness, and signs of meningeal irritation were positive. She had walking difficulty and left-sided ataxia. The rest of her general physical examination was unremarkable. The results of a blood investigation were within normal ranges (C-reactive protein $1.1 \mathrm{mg} / \mathrm{L}$; erythrocyte sedimentation rate (ESR) $15 \mathrm{~mm} / \mathrm{h}$ ). Lumbar puncture was performed, cerebrospinal fluid (CSF) cytochemical analysis findings were as follows; white blood cells $1200 / \mathrm{mm}^{3}$ (polymorphonuclear cells and lymphocytes were both seen), red blood cells $116 / \mathrm{mm}^{3}$, protein $70 \mathrm{mg} / \mathrm{dL}$, glucose $63 \mathrm{mg} / \mathrm{dL}$ (at the same time the blood glucose was $75 \mathrm{mg} / \mathrm{dL}$ ). CSF analyses performed using polymerase chain reaction (PCR) assay for herpes simplex virus type 1-2, human herpesvirus type 6-7, varicella-zoster virus (VZV), parechovirus, and enterovirus were negative. Serology of VZV, EpsteinBarr virus, cytomegalovirus, toxoplasmosis, parvovirus b19, and mycoplasma pneumonia were tested. Ceftriaxone and acyclovir treatment and dexamethasone was started for presumed infectious meningitis. She had nausea, vomiting, and diarrhea, so she was rehydrated.

Ophthalmologic consultation and fundoscopic examination was normal except for bilateral $6^{\text {th }}$ cranial nerve palsy. Brain and spine magnetic resonance imaging (MRI) scans showed normal results. An electroencephalogram revealed no abnormalities. Three days after admission, she had normal oculomotor movements and had no fever, vomiting, and diarrhea. She had mild leftsided ataxia, which resolved within two weeks.

The serology of the above mentioned microorganisms were negative except for serum mycoplasma pneumonia. Immunoglobulin (Ig)-M was borderline positive and clarithromycin treatment was added. National arbovirus and viral zoonotic diseases laboratory test results for WNV reverse transcription (RT)-PCR was negative, immune fluorescent agglutination (IFA) IgM and IgG were positive.
Serological testing of serum and cerebrospinal fluid (CSF) remains the gold standard for the WNV diagnosis. ${ }^{4}$ The result of positive IFA IgM for WNV, clinical meningitis findings with a history of being bitten by mosquitoes verified the diagnose of WNV. In the region of Esenler, where she had a history of being bitten by mosquitos adult WNV cases had previously been seen.

WNV IgM antibodies are detectable in most patients within 3 to 8 days of symptom onset and IgG antibodies are also detectable shortly after IgMantibodies. ${ }^{5}$ TheWNV serology of the patient was performed 4 days after symptom onset and both IgM and IgG antibodies were detected. Plaque reduction neutralization assay (PRNA) could be an additional diagnostic modality for this patient because flaviviruses might elicit cross-reactive test results. As a limitation PRNA could not be performed because of her parents' incompatibility. Antibiotherapy was stopped on the 10th day and she was discharged. Seven days after discharge her follow-up neurologic and systemic re-examinations were normal.

Written informed consent was obtained from the parents of the patient.

\section{Discussion}

WNV neuroinvasive infection has been defined as an illness with evidence of an acute infectious process with clinical evidence of meningitis, encephalitis or acute flaccid paralysis. ${ }^{4}$ For a definitive diagnosis of WNV infection, specific antibodies should be detected in serum or CSF samples. Detection of WNV IgM in CSF is diagnostic of neuroinvasive disease. RTPCR is not recommended for the diagnosis of WNV infection because peak viremia occurs 3-4 days before symptom onset and WNV RNA is no longer detectable. ${ }^{5}$ RT-PCR is only recommended for immunocompromised patients. ${ }^{5}$ Pleocytosis with a predominance of polymorphonuclear cells, and the presence of abnormal appearing reactive lymphocytes and elevated protein with normal glucose are the 
characteristic CSF findings of neuroinvasive WNV. ${ }^{6}$ In our patient, there were signs of meningeal irritation and CSF analysis revealed pleocytosis with polymorphonuclear cells and lymphocytes. RT-PCR was negative because peak viremia had ended. Detection of WNV IgM was diagnostic for this patient.

Serologic studies showed that WNV cases are $80 \%$ asymptomatic. ${ }^{7}$ Only $10 \%$ of symptomatic WNV cases develop neuroinvasive disease. WNV infection with neuroinvasive involvement causes encephalitis (50\%), meningitis (37\%), and acute flaccid paralysis $(6 \%) .{ }^{8}$ Also, movement disorders such as tremor, ataxia, and myoclonus may be present during WNV illness. ${ }^{9}$

In the literature, there are only two pediatric case reports describing ataxia in neuroinvasive WNV infection. ${ }^{10,11}$ A 43-year-old adult was reported with WNV who presented with ataxia and ocular dysmetria, which was reminiscent of Bickerstaff's encephalitis, and was treated with high-dose corticosteroids and intravenous immunoglobulin. ${ }^{12}$ There are no pediatric WNV case reports presenting with ataxia and ophthalmoplegia. In our case, we did not consider the diagnosis of Miller Fisher syndrome or Bickerstaff's encephalitis because of the accompanying fever, rash and CSF pleocytosis. CSF analysis and serology results excluded other viral etiologies for the differential diagnosis.

Age and sex play a role in disease severity. Older males are at greater risk for developing neuroinvasive involvement, people of younger age are more likely to present with milder forms or asymptomatic infection. ${ }^{13}$ Prognostic data on WNV neuroinvasive disease are scarce, especially for children because of the limited pediatric WNV cases. In adult studies, almost all mortality is confined to patients with neuroinvasive disease. The death rate is approximately $9 \%$ in neuroinvasive WNV cases and in patients with WNV encephalitis it is approximately $12-15 \% .{ }^{14}$ It has been shown that sequelae from neuroinvasive WNV are highly variable and younger age at infection is the most important predictor of recovery. ${ }^{15}$ In our case, the patient made a complete recovery, and we think that children with neuroinvasive WNV infections have a good prognosis.

To the best of our knowledge, this is the first published report of neuroinvasive WNV infection presenting with ophthalmoplegia. It is therefore essential for pediatricians to be familiar with the wide spectrum of neurologic manifestations of WNV disease.

\section{Author contribution}

The authors confirm contribution to the paper as follows: study conception and design: SA, GA; data collection: $\mathrm{HÖ}$; analysis and interpretation of results: HNSD; draft manuscript preparation: SA, GA. All authors reviewed the results and approved the final version of the manuscript.

\section{Conflict of interest}

None.

\section{REFERENCES}

1. Duggal NK, Langwig KE, Ebel GD, Brault AC. On the fly: interactions between birds, mosquitoes, and environment that have molded West Nile virus genomic structure over two decades. J Med Entomol 2019; 56: 1467-1474.

2. ECDC. Epidemiological update: West Nile virus transmission season in Europe, 2018. European Centre for Disease Prevention and Control, Available at: https://www.ecdc.europa.eu/en/newsevents/epidemiological-update-west-nile-virustransmission-season-europe-2018 (Accessed on October 24, 2019).

3. Hayes EB, Sejvar JJ, Zaki SR, Lanciotti RS, Bode AV, Campbell GL. Virology, pathology, and clinical manifestations of West Nile virus disease. Emerg Infect Dis 2005; 11: 1174-1179.

4. Sejvar JJ, Haddad MB, Tierney BC, et al. Neurologic manifestations and outcome of West Nile virus infection. JAMA 2003; 290: 511-515.

5. American Academy of Pediatrics. In: Kimberlin DW Brady MT, Jackson MA, Long SS, (eds). Red Book: 2018-2021 Report of the Committee on Infectious Diseases. (31st ed) Itasca, IL: American Academy of Pediatrics, 2018. 
6. Tyler KL, Pape J, Goody RJ, Corkill M, KleinschmidtDeMasters BK. CSF findings in 250 patients with serologically confirmed West Nile Virus meningitis and encephalitis. Neurology 2006; 66: 361-365.

7. Lindsey NP, Staples JE, Lehman JA, Fischer M; Centers for Disease Control and Prevention (CDC). Surveillance for human West Nile virus disease -United States, 1999-2008. MMWR Surveill Summ 2010; 59: 1-17.

8. Sejvar JJ, Marfin AA. Manifestations of West Nile neuroinvasive disease. Rev Med Virol 2006; 16: 209224.

9. Lenka A, Kamat A, Mittal SO. Spectrum of movement disorders in patients with neuroinvasive West Nile virüs infection. Mov Disord Clin Pract 2019; 6: 426-433.

10. DeBiasi RL, Parsons JA, Grabert BE. West Nile virus meningoencephalitis in an immunocompetent adolescent. Pediatr Neurol 2005; 33: 217-219.
11. Moon TD, Nadimpalli A, Martin EB, Ortiz MA, Van Dyke RB. Balance and gait abnormalities of a child with West Nile virus infection. Pediatr Infect Dis J 2005; 24: 568-570.

12. Popescu A, Jain S, Massier A, Gopalakrishna KV, Bambakidis P. West Nile infection presenting with ocular dysmetria, ataxia, and hyperreflexia reminiscent of Bickerstaff's encephalitis. Am J Med 2008; 121: e3-e4.

13. Petersen LR, Brault AC, Nasci RS. West Nile virus: review of the literature. JAMA 2013; 310: 308-315.

14. CDC. Centers for Disease Control and Prevention. Available at: http://www.cdc.gov (Accessed on November, 2020).

15. Ali M, Safriel Y, Sohi J, Llave A, Weathers S. West Nile virus infection: MR imaging findings in the nervous system. AJNR Am J Neuroradiol 2005; 26: 289-297. 